\title{
Termo-coronário-angiografia: padronização do método e primeiras aplicações clínicas no Brasil
}

\author{
Marcos Leal BRIOSCHI *,**, Mário CIMBALISTA JR. ${ }^{* *}$, Daniel COLMAN ${ }^{\star \star *}$, Tiago Noguchi MACHUCA*, \\ Danton Richlin Rocha LOURES*
}

RBCCV 44205-580

Brioschi M L, Cimbalista Jr. M, Colman D, Machuca T N, Loures D R R - Termo-coronárioangiografia: padronização do método e primeiras aplicações cirúrgicas no Brasil. Rev Bras Cir Cardiovasc 2002; 17(2): 15-19.

RESUMO: Os autores descrevem a experiência com a implantação da metodologia da termocoronário-angiografia (TCA) nos Serviços de Cirurgia Cardiovascular do Hospital de Clínicas da Universidade Federal do Paraná e Hospital Evangélico de Curitiba. Consiste em um método que permite avaliar o fluxo coronariano à distância e de maneira totalmente não-invasiva, durante a operação de revascularização miocárdica. A TCA possibilitou uma avaliação pré e pós-enxerto, com a visualização e documentação da área isquêmica na parede do miocárdio, a verificação e documentação da patência do enxerto e da anastomose, incluindo estenoses e a análise da perfusão miocárdica através dos ramos colaterais estabelecidos. A TCA é de grande auxílio para aumentar a taxa de sucesso cirúrgico e a qualidade de final de atendimento ao paciente coronariopatia. Em virtude de suas inúmeras vantagens, é forte candidata a se tornar ferramenta indispensável para uma revascularização miocárdica segura. Além disso, com um sistema de TCA instalado (permanentemente) na sala de operação cardíaca, os benefícios deste método podem ser estendidos a outros procedimentos cirúrgicos sobre o coração.

DESCRITORES: Revascularização miocárdica, métodos. Procedimentos cirúrgicos cardíacos, métodos.

\section{INTRODUÇÃO}

Em 1971, SENYK et al. (1) observaram a diminuição da temperatura da superfície miocárdica após a ligadura da artéria descendente anterior e da artéria circunflexa em cães. Estes autores também observaram uma reação hiperêmica após a reperfusão. Alguns anos depois, ROBICSEK et al. (2) descobriram que a radiação infravermelha emitida pelo coração era diretamente proporcional a sua temperatura, que por sua vez era diretamente proporcional ao fluxo coronariano. TZIVONI et al. (3) compararam alterações eletrocardiográficas aos termogramas, obtidos durante oclusões parciais ou totais da artéria descendente anterior, em cães. Com este estudo, observou-se que uma redução em $25 \%$ do fluxo coronariano não afeta a temperatura epicárdica nem o eletrocardiograma. Entretanto, reduções de $50-100 \%$ diminuíram a temperatura de superfície e renderam supradesnivelamento de segmento ST no eletrocardiograma.

O elevado fluxo sangüíneo e considerável atividade metabólica fazem do miocárdio um excelente órgão para exame por imagem infravermelha. O termo

Trabalho realizado nos Serviços de Cirurgia Cardiovascular do Hospital de Clínicas da Universidade Federal do Paraná e Hospital Evangélico de Curitiba. Curitiba, PR, Brasil.

Recebido para publicação em dezembro de 2001.

*Da Universidade Federal do Paraná.

**Da Pontifícia Universidade Católica do Paraná.

***Da Santa Casa de Misericórdia de Curitiba.

Endereço para correspondência: Marcos Leal Brioschi. Rua Cândido Mader, 74. Juvevê. Curitiba, PR. CEP: 80030-300. Tel. (41) 352-7900. e-mail: mbrioschi@ hotmail.com 
utilizado para a avaliação da temperatura da superfície cardíaca é termo-coronario-angiografia (TCA). Esta é uma ferramenta muito útil na monitoração da perfusão coronária através das diferenças de emissão de calor pelo epicárdio. Elevada emissão de calor indica um aumento do fluxo sangüíneo, enquanto que emissão diminuída indica hipoperfusão.

O objetivo deste estudo é descrever a experiência dos Serviços Universitários de Cirurgia Cardiovascular do Hospital de Clínicas da Universidade Federal do Paraná e do Hospital Evangélico de Curitiba, com a implantação da metodologia da termo-coronárioangiografia.

\section{CASUÍSTICA E MÉTODOS}

Entre junho e setembro de 2001, foram avaliados 6 pacientes do sexo masculino, com faixa etária média de 62 anos. Quatro apresentavam angina estável, um apresentava angina instável e um, infarto recente do miocárdio. Todos tinham comprometimento de pelo menos dois vasos coronarianos e foram submetidos a enxertos tanto de artéria torácica interna quanto de veia safena magna, dependendo do caso.

Foi utilizada uma câmera Thermovision AGEMA550 (FLIR SystemsÔ, Suécia) para detecção da imagem infravermelha. Esta possui um detector PtSi de resfriamento com nitrogênio líquido (steerling cicle) que atua na faixa espectral de ondas eletromagnéticas entre 3,6 e $5 \mathrm{~mm}$, o que corresponde à faixa do infravermelho médio. Sua resolução espacial máxima é de $0,1 \mathrm{a} 0,2 \mathrm{~mm}$ e a resolução térmica é de $0,1^{\circ} \mathrm{C}$. O número de pixels por imagem, isto é, pontos térmicos calibrados, é de 76.800 .

A câmera foi montada em um suporte vertical, focada diretamente na superfície exposta do coração de modo a formar um determinado ângulo com esta. O conjunto câmera-suporte foi coberto com campos cirúrgicos e transportado para trás da cabeceira do paciente. Os ajustes para a câmera foram feitos de acordo com as temperaturas medidas intraoperatoriamente pelo operador. Considerou-se para o epicárdio uma emissividade de 0,99, ou seja, $99 \%$ da radiação é emitida ao meio ambiente, não sofrendo reflexão para sua própria superfície (4).

As imagens obtidas foram exibidas em cores em um monitor de vídeo posicionado, estrategicamente, à frente do cirurgião. Cada cor representa uma isoterma. A câmera infravermelha capta as temperaturas num sistema de planilha numérica que é convertida instantaneamente, por um software, em imagem térmica, que pode ser demonstrada na forma de diversas escalas colorimétricas (palletes) de acordo com a melhor definição e contraste para determinada situação. As imagens selecionadas foram realçadas e analisadas utilizando-se um processador de imagens específico (ThermaCAMÔ Researcher 2001, FLIR Systems, Suécia). As imagens finais foram armazenadas em um microcomputador Pentium III acoplado a uma placa PCMCIA e também gravadas em uma fita de videocassete.

Após a anastomose venosa distal, $20 \mathrm{ml}$ de soro fisiológico resfriado $\left(8-14^{\circ} \mathrm{C}\right)$ foram injetados em bolo para sua visualização. Foram obtidas imagens em intervalos de $1 / 7$ segundo para verificar a integridade da anastomose e visibilizar a anatomia coronariana distal (cine-termo-coronario-angiografia). Após a anastomose proximal, dois termogramas foram obtidos antes de liberar o fluxo sangüíneo através da ponte aorto-coronária. Após sua liberação, novas imagens foram obtidas em intervalos de 1/7 segundos.

No caso dos enxertos arteriais, as imagens foram obtidas de forma semelhante, após a retirada da pinça hemostática da artéria torácica interna, permitindo a restauração do fluxo com sangue aquecido $\left(25\right.$ a $\left.30^{\circ} \mathrm{C}\right)$.

As temperaturas retal e orofaríngea foram também obtidas antes da restauração do fluxo e ao final do período de observação.

\section{RESULTADOS}

Em todos os pacientes, a equipe de cirurgia cardíaca obteve imagens que proporcionaram a perfeita visibilização do fluxo sangüíneo no enxerto, na artéria coronária e no local da anastomose. Foi possível a visibilização de ramos de até $4^{\mathrm{a}}$ ordem a partir do tronco coronariano principal. Além disso, pode-se avaliar em todos os casos, também pela TCA, a viabilidade miocárdica após a reperfusão.

Para compreender toda área de interesse, a focalização foi obtida com uma lente de germânio de 10 . A distância mínima entre a câmera e o campo operatório para obtenção do foco da imagem térmica foi superior a 1,3 metro. A câmera foi ajustada de modo a formar um ângulo de no mínimo $45^{\circ} \mathrm{com}$ a superfície do coração (Figura 1).

As escalas colorimétricas (palletes) mais adequadas foram a glowbow e a midgray. A escala contínua glowbow, que representa as temperaturas da mais quente para a mais fria respectivamente nas cores branco, laranja e preto, permitiu o melhor contraste durante a avaliação da permeabilidade do enxerto coronariano. Já a escala contínua midgray (azul para o mais frio e o vermelho para o mais quente) permitiu o melhor contraste para a avaliação da perfusão do epimiocárdio.

O ajuste necessário na escala térmica para obtenção das imagens foi obtido com uma amplitude térmica (range) entre $25,6^{\circ}-38,8^{\circ} \mathrm{C}$ e temperatura média (level) de $32,2^{\circ} \mathrm{C}$.

A parede posterior foi visualizada após manobra de semi-rotação do coração de LIMA ${ }^{(5)}$ (Figura 2). 


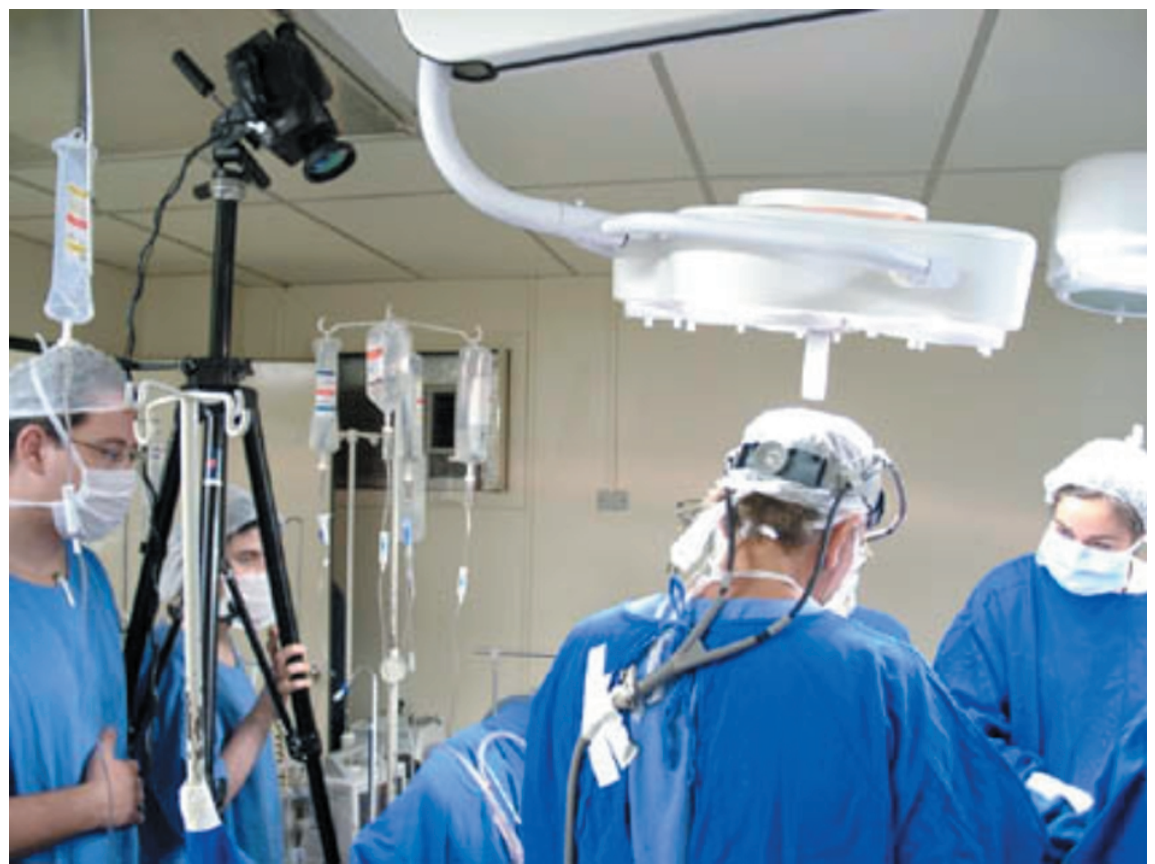

Fig. 1 Posicionamento da câmera infravermelha a uma distância de 1,3 metro do campo operatório.

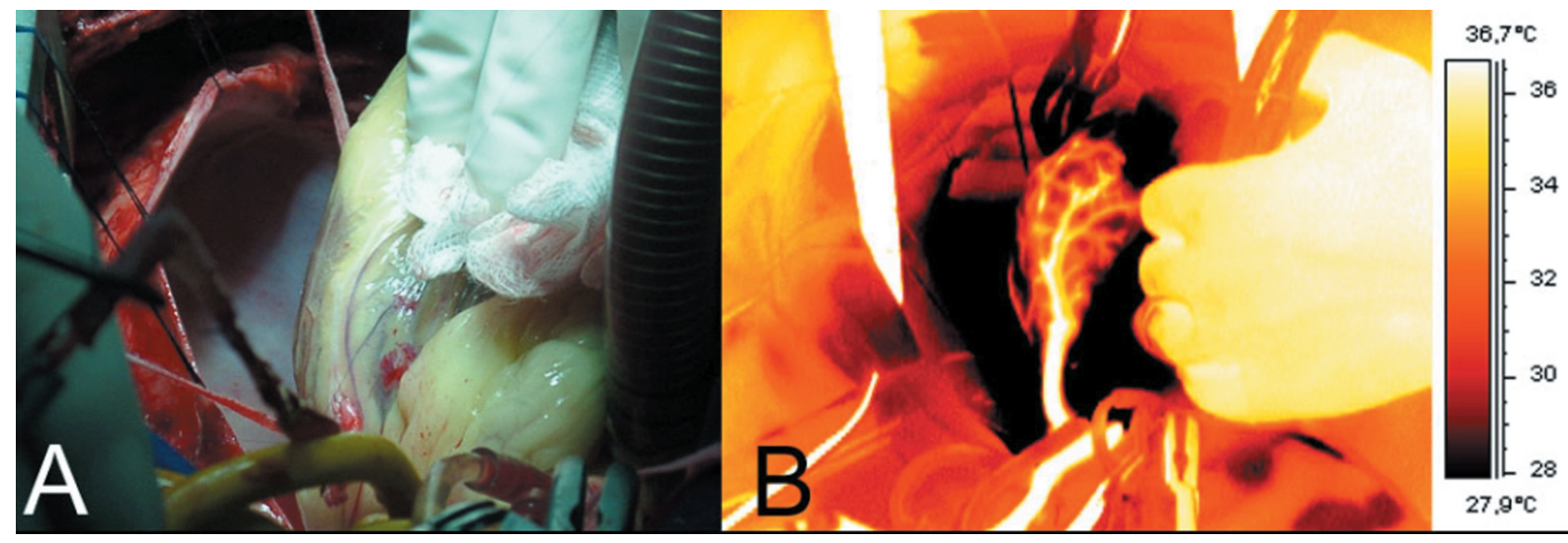

Fig. 2 A) Visualização da ponte de safena magna na porção posterior da artéria circunflexa após manobra de Lima.

B) Imagem térmica infravermelha correspondente a figura A mostrando a permeabilidade da artéria circunflexa e a perfusão na parede posterior pelos seus ramos até $4^{a}$ ordem. (escala colorimétrica glowbow).

A cine-termo-coronario-angiografia foi obtida com a gravação em tempo real de 7 imagens por segundo, permitindo assim a análise da reperfusão.

As imagens da artéria coronária reperfundida puderam ser ampliadas até 4 vezes, proporcionando uma avaliação detalhada do diâmetro do vaso.
A variação de temperatura da superfície miocárdica é limitada a $30-37^{\circ} \mathrm{C}$, enquanto que a das artérias coronárias sem resfriamento por circulação extracorpórea é de $34-35^{\circ} \mathrm{C}$, aproximadamente.

Inicialmente, a temperatura da superfície do músculo cardíaco durante a anastomose do enxerto, 
usando o método da cardioplegia, caiu a $23 \pm 2^{\circ} \mathrm{C}$. Gradualmente, esta aumentou para $27^{\circ} \mathrm{C}$. Após a remoção das pinças hemostáticas, imagens da elevada temperatura da artéria coronária foram visualizadas em regiões de seu leito distal.

A viabilidade do músculo cardíaco foi avaliada através da comparação das temperaturas obtidas antes e depois da restauração do fluxo sangüíneo.

A TCA possibilitou uma avaliação pré e pós-enxerto. No tempo pré-enxerto foi possível:

- Localizar as artérias coronárias nativas;

- Visualizar e documentar a área isquêmica na parede do miocárdio;

- Visualizar e documentar a perfusão da cardioplegia, assegurando assim a proteção miocárdica;

- Auxiliar a localização de artérias coronárias nos casos de reoperação.

No tempo pós-enxerto foi possível, mesmo em parede posterior:

- Verificar e documentar a patência do enxerto, incluindo estenoses;

- Verificar e documentar a perfusão miocárdica através dos ramos colaterais estabelecidos;

- Verificar e documentar os sentidos de fluxo, retrógrado ou anterógrado;

- Visualizar e documentar o estado da anastomose, incluindo estenoses;

- Visualizar e documentar o fluxo sangüíneo distal à anastomose para checar oclusões distais ao local da ponte.

\section{COMENTÁRIOS}

Uma vez que a injeção de solução cardioplégica resfriada e a liberação das pinças hemostáticas no enxerto venoso ou arterial são manobras de rotina na operação de revascularização, a TCA apenas necessitou de uma boa exposição do órgão e obtenção de foco pela câmera, não representando qualquer tipo de interferência no ato cirúrgico. A diferença de temperatura entre o líquido da artéria coronária e a superfície epicárdica rendeu imagens que propiciaram o delineamento da anatomia coronariana e do enxerto.

TCA é um dos métodos mais precisos para estimar intra-operatoriamente o fluxo coronariano durante a operação de revascularização do miocárdio. Nenhum tipo de cateter, meio de contraste ou radiação se faz necessário. FALK et al. ${ }^{(6)}$ utilizaram este método em 370 pacientes, obtendo sucesso na visualização do fluxo em $90,6 \%$ dos enxertos venosos e em $96,2 \%$ dos enxertos com a artéria torácica interna. MERIN et al. ${ }^{(7)}$ relataram a detecção intraoperatória de lesões no leito coronário distal até então desconhecidas. Entretanto, a TCA ainda não é uma técnica de uso rotineiro. Uma vez que a operação de revascularização do miocárdio é um procedimento bem estabelecido, quase todos os cirurgiões cardíacos têm se baseado na sua experiência pessoal através de injeções manuais de líquido através da veia safena magna ou, quando o enxerto é arterial, somente na coloração da superfície cardíaca e na ausência de instabilidade hemodinâmica para avaliar a viabilidade miocárdica. Outros métodos para mensuração do fluxo coronariano também não são rotineiramente empregados.

Ao contrário do que ocorre com a superfície corporal e pele, os órgãos internos não são envoltos por um isolante térmico. De acordo com a terceira lei de Newton, eles resfriam uma vez expostos a ambientes resfriados. A temperatura é determinada basicamente pelo fluxo do sangue que perfunde 0 órgão ${ }^{(8)}$. Por esta razão, a viabilidade do músculo cardíaco pode ser avaliada pelo registro contínuo de imagens térmicas do coração. Caso exista uma rede arterial em determinado órgão e o sangue aquecido supre esta rede, o seu padrão anatômico é claramente observado em imagens térmicas deste órgão. A rede arterial coronariana existe na superfície cardíaca (epimiocárdio). Quando a pinça hemostática é liberada após a realização da anastomose, pode-se observar o padrão do sangue aquecido fluindo pelos ramos das artérias coronárias ${ }^{(9-12)}$.

\section{CONCLUSÕES}

O fluxo coronariano pode ser observado a distância e de maneira totalmente não-invasiva com a TCA. Esta é de grande auxílio para aumentar a taxa de sucesso cirúrgico e a qualidade final de atendimento ao paciente coronariopata. Em virtude de suas inúmeras vantagens, é forte candidata a se tornar ferramenta indispensável para uma revascularização miocárdica segura. Além disso, com um sistema de TCA instalado (permanentemente) na sala de operação cardíaca, os benefícios deste método podem ser estendidos a outros procedimentos sobre o coração. 
RBCCV 44205-580

Brioschi ML, Cimbalista Jr. M, Colman D, Machuca T N, Loures D R R - Thermo-coronaryangiography: standardization of the method and first clinical applications in Brazil. Rev Bras Cir Cardiovasc 2002; 17(2): 14-17.

ABSTRACT: The authors report the experience with the methodological implantation of thermocoronary-angiography in the Division of Cardiovascular Surgery of the Hospital de Clínicas, Federal University of Paraná, and the Hospital Universitário Evangélico de Curitiba. It is a totally noninvasive technique that allows the real time assessment of the coronary blood flow with no interruption on the surgical procedure. Using TCA, it was possible to analyze the heart before and after the bypass graft completion, which allowed the visualization and recording of the ischemic area in the myocardial wall, the assessment of the graft patency, including stenoses, and the status of myocardial perfusion by preestablished collateral branches. Therefore, TCA is helpful in order to improve the results of the coronary artery bypass graft surgery, providing a higher treatment quality to the patient with coronary artery disease. Due to its great number of advantages, this infrared imaging method has an enormous likelihood to become a crucial tool to perform a safe myocardial revascularization. Further more, a permanent TCA system established in the operating room would extend all its benefits to other cardiac surgical procedures.

DESCRIPTORS: Myocardial revascularization, methods. Cardiac surgical procedures, methods.

\section{REFERÊNCIAS BIBLIOGRÁFICAS}

1. Senyk J, Malm A, Bornmyr S - Intraoperative cardiothermography: a new method for detecting ischemic areas in the heart muscle and for investigating the results of revascularization procedures in coronary surgery. Eur Surg Res 1971; 3: 1-12.

2. Robicsek F, Masters TN, Svenson RH et al. The application of thermography in the study of coronary blood flow. Surgery 1978; 84: 858-64.

3. Tzivoni D, Cribier A, Kanmatsuse K, Chew C, Ganz W - Evaluation of changes in epicardial blood flow in experimental animals by cardiothermography. Eur Heart J 1982; 3: 382-8.

4. Szabó T, Fazekas L, Gellér L et al. Cardiothermographic assessment of arterial and venous revascularization. IEEE Eng Med Biol Mag. 2000; 19: 77-82.

5. Cohn WE, Weintraub RM, Sellke FW Innovative minimally invasive surgical approaches to coronary revascularization in the high risk patient. Heart Surg Forum 2000; 3: 185-8.

6. Falk V, Walther T, Philippi A et al. - Thermal coronary angiography for intraoperative patency control of arterial and saphenous vein coronary artery bypass grafts: results in 370 patients. J Card Surg 1995; 10: 147-60.
7. Merin G, Elami A, Zucker M - Intraoperative detection of unsuspected distal coronary obstruction by thermal coronary angiography. Cardiovasc Surg 1995; 3: 599- 601.

8. Brioschi ML, Cimbalista Jr M, Nakagawa CR, von Bahten LC, Brofman PS - Avaliação intraoperatória da revascularização cardíaca por angiografia térmica coronária: estudo experimental preliminar. Arq Med PUCPR 2000; 2.

9. Mohr FW, Matloff J, Grundfest W et al. - Thermal coronary angiography: a method for assessing graft patency and coronary anatomy in coronary bypass surgery. Ann Thorac Surg 1989; 47:441-9.

10. Mohr FW, Falk V, Krieger $\mathrm{H}$ et al. - IMA-graft patency control by thermal coronary angiography during coronary bypass surgery. Eur $J$ Cardiothorac Surg 1991; 5: 534-41.

11. Fujimasa I, Nakazawa H, Kawada S - A new thermal coronary angiography system. Thermol Int 2001; 11: 25-8.

12. Suma $H$, Isomura $T$, Horii $T$, Sato $T$ Intraoperative coronary artery imaging with infrared camera in off-pump CABG. Ann Thorac Surg 2000; 70: $1741-2$. 